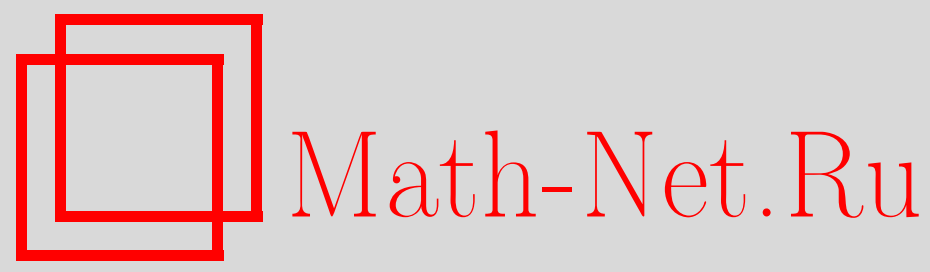

Г. Е. Пунинский, Левые почти расщепляющиеся морфизмы и обобщенные алгебры Вейля, Матем. заметки, 1999, том 66, выпуск 5, 734-740

DOI: https://doi.org/10.4213/mzm1217

Использование Общероссийского математического портала Math-Net.Ru подразумевает, что вы прочитали и согласны с пользовательским соглашением http://www . mathnet.ru/rus/agreement

Параметры загрузки:

IP : 52.23 .180 .231

26 апреля 2023 г., 07:41:06

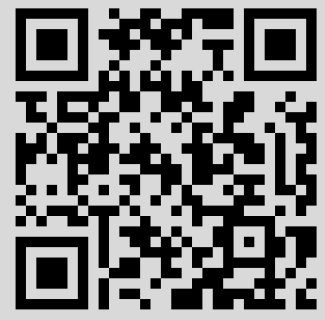




\title{
ЛЕВЫЕ ПОЧТИ РАСЩЕПЛЯЮЩИЕСЯ \\ МОРФИЗМЫ И ОБОБЩЕННЫЕ АЛГЕБРЫ ВЕЙЛЯ
}

\section{Г.Е. Пунинский}

\begin{abstract}
Доказьвается, что категория модулей конечной длины над широким классом обобщенных алгебр Вейля не содержит ни одного левого почти расщепляющегося морфизма, начинающегося с простого модуля. Показано, что аналогичный факт имеет место для алгебры $U \mathrm{sl}_{2}(\mathbf{k})$ над алгебраически замкнутым полем $\mathbf{k}$ характеристики 0 . Попутно строится новая серия простых модулей для таких алгебр.
\end{abstract}

Библиография: 5 названий.

1. Введение. Понятие обобщенной алгебры Вейля неявно использовалось многими авторами, и было четко сформулировано в работе Бавулы [1]. Теория таких алгебр и модулей над ними достаточно полно исследована (см. [2] для подробной информации и библиографии). Например, в [1], [2] приведена классификация простых модулей над обобщенной алгеброй Вейля в случае, когда базисное кольцо является коммутативной дедекиндовой областью.

Почти расщепляющиеся последовательности были введены Ауслендером и играют значительную роль в теории представлений артиновых алгебр. Для других некоммутативных колец это понятие мало исследовано. В работе [3] Циммерман показал, что категория модулей конечной длины над некоторьми кольцами дифференциальных многочленов обладает почти расщепляющимися последовательностями. Из результатов Преста и Пунинского [4] вытекает, что над первой алгеброй Вейля над полем характеристики нуль категория модулей конечной длины не имеет ни одной почти расщепляющейся последовательности. Там же была выдвинута гипотеза, что ни один модуль конечной длины над обобщенной алгеброй Вейля без циклических орбит не является началом левого почти расщепляющегося морфизма.

В настоящей заметке мы проверим эту гипотезу в случае простого модуля над обобщенной алгеброй Вейля такой, что любая орбита линейна и содержит не более трех корней определяющего многочлена (без учета кратности). Как следствие мы докажем, что это верно для алгебры $U \mathrm{sl}_{2}(\mathbf{k})$, где $\mathbf{k}$ - алгебраически замкнутое поле характеристики нуль.

Работа выполнена при частичной поддержке Российского фонда фундаментальных исследований. 
Основное техническое средство, которое мы используем, - классификация простых модулей над обобщенной алгеброй Вейля [1] и техника вычислений модуля расширений [4], [5]. Реализуя этот подход, мы строим новую серию простых модулей без $\mathbf{k}[H]$-кручения, отталкиваясь от самого левого корня определяющего многочлена на фиксированной линейной орбите. Например, это дает новую серию простых модулей над алгеброй $U \mathrm{Sl}_{2}(\mathbf{k})$.

2. Определения. На протяжении этой работы пусть $\mathbf{k}$-поле характеристики нуль, $\mathbf{k}^{*}=\mathbf{k} \backslash 0$ - его мультипликативная группа, $\mathbf{k}[H]$ - кольцо многочленов над $\mathbf{k}$ и $\sigma-$ автоморфизм кольца $\mathbf{k}[H]$. Алгебра $A$ над $\mathbf{k}$ назьвается обобщенной алгеброй Вейля (OAB) с определяющим многочленом $a(H) \neq 0$, если $A$ порождена над $\mathbf{k}[H]$ переменньми $X, Y$ с определяющими соотношениями

$$
Y X=a(H), \quad X Y=\sigma(a), \quad b(H) Y=Y \sigma(b) \quad \text { и } \quad b(H) X=X \sigma^{-1}(b)
$$

для произвольного $b(H) \in \mathbf{k}[H]$. Учитьвая эти соотношения, любой элемент $u \in A$ может быть однозначно записан в виде

$$
u=Y^{m} a_{-m}(H)+\cdots+a_{0}(H)+\cdots+X^{n} a_{n}(H)
$$

или в виде

$$
u=\sigma^{-m}\left(a_{-m}\right) Y^{m}+\cdots+a_{0}(H)+\cdots+\sigma^{n}\left(a_{n}\right) X^{n} .
$$

Например (см. [1]), первая алгебра Вейля $A_{1}$ над полем $\mathbf{k}$ является ОАВ с $a(H)=H$ и $\sigma(H)=H-1$. Для универсальной обертывающей алгебры $U \operatorname{sl}_{2}(\mathbf{k})=\langle X, Y, H|$ $[H, X]=X,[H, Y]=-Y,[X, Y]=2 H\rangle$ с элементом Казимира $C=H(H+1)+Y X$ и $\lambda \in \mathbf{k}$ ее фактор-алгебра $A_{\lambda}=U \mathrm{sl}_{2}(\mathbf{k}) / U \mathrm{sl}_{2}(\mathbf{k})(C-\lambda)$ также является обобщенной алгеброй Вейля с $\sigma(H)=H-1$ и $a(H)=\lambda-H(H+1)$.

Каждьй максимальный идеал кольца $\mathbf{k}[H]$ порожден неразложимым многочленом $p(H)$, поэтому правило $p \rightarrow \sigma(p)$ задает действие $\sigma$ на множестве максимальньх идеалов $\mathbf{k}[H]$. Каждая орбита этого действия есть либо иикл, либо иепь, т.е. вьглядит как линейный порядок целых чисел $\mathbb{Z}$. В силу [1, теорема 2.1] любая ОАВ без циклов $A$ является нётеровой областью размерности Крулля 1 , т.е. любой собственньй фактор-модуль алгебры $A$ имеет конечную длину.

Классификация простых модулей над ОАВ $A$ содержится в [2]. В частности, любой простой модуль над $A$ либо является $\mathbf{k}[H]$-модулем кручения, либо не имеет $\mathbf{k}[H]$-кручения. Предположим, что $\sigma$ не имеет циклических орбит. Простые модули с $\mathbf{k}[H]$-кручением описьваются в терминах разбиения орбит действия $\sigma$ делителями $a(H)$ вида $\sigma^{n}(p)$. Например, если для неразложимого полинома $p$ имеем $p(H), \sigma^{n}(p)$ делят $a(H)$ и $\sigma^{m}(p)$ не делит $a(H)$ для всех $0<m<n$, то модуль $A / A\left(X, Y^{n}, \sigma^{n}(p)\right)$ прост и конечномерен над $A$, и все простые конечномерные модули над $A$ получаются таким образом.

В ОАВ $A$ множество $S=\mathbf{k}[H] \backslash 0$ есть левое и правое множество Оре, и кольцо частных $B=S^{-1} A$ является кольцом косых многочленов Лорана $B=\mathbf{k}\left[X, X^{-1}, \sigma\right]$, т.е. $B$ - область главных левых и правых идеалов. Пусть $p \in A$ - неразложимьй в $B$ элемент, который действует левьп умножением как мономорфизм на любом простом $A$-модуле с 
$\mathbf{k}[H]$-кручением. Тогда ввиду $[2$, лемма 5.7, следствие 5.4$]$ имеет место короткая точная последовательность

$$
0 \rightarrow V_{p}=A \cap B p / A p \rightarrow A_{p}=A / A p \rightarrow M_{p}=A / A \cap B p \rightarrow 0
$$

где $M_{p}$ - простой $A$-модуль, а $V_{p}-\mathbf{k}[H]$-модуль кручения.

3. Простые модули. Пусть $p(H)$ неразложимый многочлен, причем $p(H) \mid a(H)$ и $\sigma^{n}(p)$ не делит $a(H)$ для целого $n<0$. Для $0 \neq \alpha \in \mathbf{k}$ положим

$$
p_{\alpha}=\alpha Y+p(H), \quad t_{\alpha}=\sigma(\alpha) \frac{\sigma(a)}{\sigma(p)}+X
$$

и $A_{\alpha}=A / A p_{\alpha}, M_{\alpha}=A / A\left(p_{\alpha}, t_{\alpha}\right)$. Поскольку $p(H) \mid a(H)$, то $t_{\alpha} \in A$.

ПРЕДЛОЖЕНИЕ 1. Модуль $M_{\alpha}, 0 \neq \alpha \in \mathbf{k}$, прост без $\mathbf{k}[H]$-кручения. Если фактор $\mathbf{k}^{*}$ по подгруппе $\left\{\sigma(\gamma) \gamma^{-1} \mid 0 \neq \gamma \in \mathbf{k}\right\}$ бесконечен, то имеется бесконечное число неизоморфных $M_{\alpha}$.

ДокАЗАтЕльство. Проверим сначала, что любой элемент $v \in A / A p_{\alpha}$ имеет каноническую форму $v=b_{0}(H)+X b_{1}(H)+\cdots+X^{n} b_{n}(H)$, где $\operatorname{deg}\left(b_{i}\right)<\operatorname{deg}(p)$ при $i \geqslant 1$. Поскольку $\alpha Y+p(H)=0$ в $A_{\alpha}$, то, домножая слева на $X$, получаем $\sigma(\alpha) \sigma(a)+$ $\sigma(p) X=0$, поэтому в $A_{\alpha}$ имеют место соотношения

$$
Y \equiv-\alpha^{-1} p(H) \quad \text { и } \sigma(p) X \equiv-\sigma(\alpha) \sigma(a) \text { или } X p \equiv-\sigma(\alpha) \sigma(a) \text {. }
$$

По модулю этих соотношений любой элемент может быть приведен к нужному виду. Если это представление не единственно, то

$$
b_{0}(H)+X b_{1}(H)+\cdots+X^{n} b_{n}(H)=\left(\cdots+X^{n} c_{n}(H)\right)(\alpha Y+p(H)),
$$

откуда (при $X^{n}$ ) получаем $b_{n}(H)=c_{n}(H) p(H)$, противоречие.

Проверим, что $\sigma(p) t_{\alpha}=Y t_{\alpha}=0$ в $A_{\alpha}$. Действительно,

$$
\sigma(p) t_{\alpha}=\sigma(\alpha) \sigma(a)+\sigma(p) X=X \cdot(\alpha Y+p(H))=X p_{\alpha}
$$

поэтому $\sigma(p) t_{\alpha}=0$ в $A_{\alpha}$. Аналогично

$$
Y t_{\alpha}=\alpha \frac{a(H)}{p(H)} Y+a(H)=\frac{a(H)}{p(H)} \cdot(\alpha Y+p(H))=\frac{a(H)}{p(H)} p_{\alpha}
$$

влечет $Y t_{\alpha}=0$ в $A_{\alpha}$. Кроме того, $t_{\alpha} \neq 0$ в $A_{\alpha}$.

Покажем теперь, что любой элемент $v \in M_{\alpha}$ имеет каноническую форму $v=u(H) \in$ $\mathbf{k}[H]$. Действительно, в $M_{\alpha}$ вьполнено соотношение

$$
X \equiv-\sigma(\alpha) \frac{\sigma(a)}{\sigma(p)}
$$


следовательно, $v$ можно привести к такому виду.

Если это представление не единственно, то $0 \neq u(H) \in A\left(p_{\alpha}, t_{\alpha}\right)$, т.е. $u=z t_{\alpha}$ в $A_{\alpha}$, где равенство должно стать тождеством послеприведения левой и правой части к канонической форме в $A_{\alpha}$. Учитьвая соотношения $\sigma(p) t_{\alpha}=Y t_{\alpha}=0$ в $A_{\alpha}$, можно привести это равенство к виду

$$
u(H)=\left(c_{0}+X c_{1}+\cdots+X^{n} c_{n}\right)\left(\sigma(\alpha) \frac{\sigma(a)}{\sigma(p)}+X\right), \quad \operatorname{deg}\left(c_{i}\right)<\operatorname{deg}(\sigma(p))
$$

Старший член в правой части есть $X^{n+1} \sigma^{-1}\left(c_{n}\right)$, причем $\operatorname{deg}\left(\sigma^{-1}\left(c_{n}\right)\right)<\operatorname{deg}(p)$ и $n+1 \geqslant 1 ;$ противоречие.

Докажем теперь простоту $M_{\alpha}$. Пусть $0 \neq u(H)$ порождает собственный помодуль в $M_{\alpha}$ и выберем такое $u$ минимальной возможной степени. Учитывая соотношения в $M_{\alpha}$, имеем

$$
Y u(H)=\sigma^{-1}(u) Y=-\alpha^{-1} \sigma^{-1}(u) p(H) .
$$

Тогда $u(H)$ делит $\sigma^{-1}(u) p(H)$. Поскольку $\sigma$ не имеет циклических орбит, $u(H)$ есть с точностью до мультипликативной константы $\sigma^{-k}(p) \cdot \ldots \cdot p$ для $k \geqslant 0$. Кроме того,

$$
X u(H)=\sigma(u) X=-\sigma(\alpha) \sigma(u) \cdot \frac{\sigma(a)}{\sigma(p)}
$$

следовательно, $u(H)$ делит последний многочлен. Сокращая, получаем, что $\sigma^{-k}(p)$ делит $\sigma(a)$; следовательно, $\sigma^{-k-1}(p)$ делит $a(H)$; противоречие.

Из канонической формы элементов $M_{\alpha}$ видно, что $M_{\alpha}$ не имеет $\mathbf{k}[H]$-кручения.

Осталось только проверить, что среди $M_{\alpha}$ имеется бесконечное число не изоморфных модулей. Предположим что $\alpha \neq \beta$ и $f: M_{\alpha} \rightarrow M_{\beta}$ ненулевой гомоморфизм. Тогда $f$ задан правым умножением на элемент $u(H) \in \mathbf{k}[H]$. Образ $p_{\alpha}$ при умножении на $u(H)$ равен нулю в $M_{\beta}$. Пересчитаем каноническую форму элемента $p_{\alpha} u$, используя соотношения (2); имеем

$$
(\alpha Y+p) u(H)=\alpha \sigma^{-1}(u) Y+p u(H)=-\alpha \beta^{-1} \sigma^{-1}(u) p+p u(H)=0 .
$$

Тогда $\alpha \beta^{-1} \sigma^{-1}(u)=u(H)$. Если $\operatorname{deg}(u) \geqslant 1$, то $\sigma$ имеет циклическую орбиту, что невозможно. Иначе $u(H)=\gamma \in \mathbf{k}$, откуда $\sigma\left(\alpha \beta^{-1}\right)=\sigma(\gamma) \gamma^{-1}$. По предположению о бесконечности фактора получаем бесконечное число неизоморфных модулей $M_{\alpha}$.

Из классификации простых $A$-модулей с $\mathbf{k}[H]$-кручением вытекает, что $p_{\alpha}, 0 \neq \alpha \in \mathbf{k}$, действует левым умножением как мономорфизм на любом таком модуле. По (1) получаем короткую точную последовательность

$$
0 \rightarrow V_{\alpha}=A \cap B p_{\alpha} / A p_{\alpha} \rightarrow A_{\alpha}=A / A p_{\alpha} \rightarrow A / A \cap B p_{\alpha} \rightarrow 0
$$

где $A / A \cap B p_{\alpha}-$ простой $A$-модуль и $V_{\alpha}-\mathbf{k}[H]$-модуль кручения. 
ЛЕмма 1. $V_{\alpha}$ порожден әлементом $t_{\alpha} u A / A \cap B p_{\alpha}=M_{\alpha}$.

ДокАЗАТЕЛьство. Действительно, из доказательства предложения 1 имеем $\sigma(p) t_{\alpha}=X p_{\alpha}$, откуда $t_{\alpha}=\sigma(p)^{-1} X p_{\alpha} \in B p_{\alpha} \cap A$, т.е. $t_{\alpha} \in V_{\alpha}$. Кроме того, $t_{\alpha} \neq 0$ в $V_{\alpha}$.

Итак, $\sigma(p) t_{\alpha}=0$ в $V_{\alpha}$. Аналогично, учитьвая равенство $Y t_{\alpha}=[a(H) / p(H)] p_{\alpha}$, получаем $Y t_{\alpha}=0$ в $V_{\alpha}$. Если $V_{\alpha}$ не порожден $t_{\alpha}$, то $A / A \cap B p_{\alpha}$ является собственным фактором простого модуля $M_{\alpha}$; противоречие.

Заметим, что для первой алгебры Вейля $A_{1}$ над полем $\mathbf{k}$ для $p(H)=H$ получаем $t_{\alpha}=\alpha+X$ и $Y t_{\alpha}=\alpha Y+H=p_{\alpha}$, т.е. $M_{\alpha}=A / A t_{\alpha}$. Нетрудно проверить, что $t_{\alpha}$ и $p_{\alpha}$ пропорциональны в $A$, если и только если $a(H)=\gamma p(H), 0 \neq \gamma \in \mathbf{k}$.

Напомним, что для элемента Казимира $C$ и $\lambda \in \mathbf{k}$ фактор-алгебра $A_{\lambda}=$ $U \mathrm{sl}_{2}(\mathbf{k}) / U \mathrm{sl}_{2}(\mathbf{k})(C-\lambda)$ является обобщенной алгеброй Вейля с $\sigma(H)=H-1$ и $a(H)=$ $\lambda-H(H+1)$. Корни $a(H)$ (в алгебраическом расширении $\mathbf{k})$ есть $\tau_{1,2}=1 / 2 \cdot(-1 \pm$ $\sqrt{1+4 \lambda})$.

ПРИмЕР 1. Пусть $\mathbf{k}$ - поле характеристики нуль, $\sqrt{1+4 \lambda} \in \mathbf{k}$ для $\lambda \in \mathbf{k}$ и $\tau \in \mathbf{k}-$ меньшее из чисел $1 / 2 \cdot(-1 \pm \sqrt{1+4 \lambda})$, если $\sqrt{1+4 \lambda} \in \mathbb{Z}$ (иначе, любое из этих чисел). Тогда

$$
M_{\lambda, \alpha}=U \operatorname{sl}_{2}(\mathbf{k}) / U \operatorname{sl}_{2}(\mathbf{k})\left(C-\lambda, p_{\alpha}, t_{\alpha}\right),
$$

где $C=H(H+1)+Y X-$ элемент Казимира,

$$
p_{\alpha}=\alpha Y+(H-\tau), \quad t_{\alpha}=-\alpha\left(H-1+\frac{\lambda}{\tau}\right)+X,
$$

$0 \neq \alpha \in \mathbf{k}$ - простые неизоморфные $U \mathrm{Sl}_{2}(\mathbf{k})$ модули без $\mathbf{k}[H]$-кручения.

4. Левые почти расщепляющиеся морфизмы. Пусть $\mathcal{A}$ - полная наследственная подкатегория категории модулей над кольцом и $f: M \rightarrow N-$ морфизм в $\mathcal{A}$. Скажем, что $f$-левый почти расщепляющийся морфизм (левьй п.р.м.), если 1) $f$ не является расщепляющимся мономорфизмом и 2) любой морфизм $g: M \rightarrow K$ в $\mathcal{A}$, не являющийся расщепляющимся мономорфизмом, пропускается через $f$, т.е. $g=f h$ для некоторого морфизма $h: N \rightarrow K$ в $\mathcal{A}$. Например, для простого инъективного модуля $M$ морфизм $f: M \rightarrow 0$ есть левьй п.р.м.

Нам потребуется следующий результат

ФАКТ $1[4$, предложения $3.7,3.8]$. Пусть $\mathcal{K}$ - категория модулей конечной длины над произвольным кольцом $и M \in \mathcal{K}$ таков, что $\operatorname{Ext}\left(M_{i}, M\right) \neq 0$ для бесконечного числа неизоморфных простых модулей $M_{i}$. Тогда не существует левого почти расщепляющегося морфизма в $\mathcal{K}$, начинающегося $с M$.

Основным результатом работы является следующая теорема.

Теорема 1. Пусть А обобщенная алгебра Вейля над полем $\mathbf{k}$ характеристики нуль, фактор $k^{*}$ по подгруппе $\left\{\sigma(\gamma) \gamma^{-1} \mid 0 \neq \gamma \in \mathbf{k}\right\}$ бесконечен $и$ для любого неразложимого полинома $p(H)$ существует самое большее три $n \in \mathbb{Z}$ таких, что $\sigma^{n}(p)$ делит $a(H)$. Тогда ни один простой модуль над $A$ не является началом левого почти расщепляющегося морфизма в категории модулей конечной длинь над $A$. 
ДокАЗАТЕЛЬСТво. По факту 1 достаточно для любого простого модуля $M$ над $A$ указать набор неизоморфных простых модулей $M_{i}$ таких, что $\operatorname{Ext}\left(M_{i}, M\right) \neq 0$. В работе [4] рассмотрены все случаи, кроме конечномерного $M$. Итак, можно считать, что $M$ конечномерен. Предположим сначала, что $M$ отвечает самому левому конечному полуинтервалу на вырожденной орбите. Тогда $M \cong A / A\left(X, Y^{n}, \sigma^{n}(p)\right)$, где $p(H), \sigma^{n}(p)$ | $a(H)$ для некоторого целого $n>0$ и $\sigma^{m}(p)$ не делит $a(H)$ для всех $m<n, m \neq 0$. Если $m=\overline{1}$ порождающий $M$, то $l=Y^{n-1} m \neq 0$ - другой порождающий, и $Y l=X^{n} l=$ $\sigma(p) l=0$ - определяющие соотношения для $M$.

Рассмотрим построенное в предложении 1 семейство модулей $V_{\alpha}, M_{\alpha}$. Поскольку $p_{\alpha}$ действует (левым умножением) как мономорфизм на любом простом модуле $\mathbf{c} \mathbf{k}[H]$-кручением и $M$ конечномерен, $p_{\alpha}$ действует как автоморфизм векторного пространства $M$, откуда $\operatorname{Ext}\left(A_{\alpha}, M\right)=0$. Применим функтор $\operatorname{Hom}(-, M)$ к точной последовательности (4). Получаем

$$
\begin{aligned}
0 & \rightarrow \operatorname{Hom}\left(M_{\alpha}, M\right) \rightarrow \operatorname{Hom}\left(A / A p_{\alpha}, M\right) \rightarrow \operatorname{Hom}\left(V_{\alpha}, M\right) \\
& \rightarrow \operatorname{Ext}\left(M_{\alpha}, M\right) \rightarrow \operatorname{Ext}\left(A / A p_{\alpha}, M\right) \rightarrow \operatorname{Ext}\left(V_{\alpha}, M\right) \rightarrow \cdots
\end{aligned}
$$

Докажем, что $\operatorname{Hom}\left(A_{\alpha}, M\right)=0$. Любой элемент $u \in M$ имеет канонический вид $u=b_{0}(H)+X b_{1}(H)+\cdots+X^{n-1} b_{n-1}(H), \operatorname{deg}\left(b_{i}\right)<\operatorname{deg}(\sigma(p))$. Пусть гомоморфизм $f: A_{\alpha} \rightarrow M$ задан правым умножением на $u$. Тогда $(\alpha Y+p(H)) u=0$ в $M$. Вьгисляя в $M$, имеем $(\alpha Y+H) u=\left(\alpha a(H) b_{1}(H)+p(H) b_{0}(H)\right)+\cdots+X^{n-2}\left(\alpha \sigma^{-n+2}(a) b_{n-1}(H)+\right.$ $\left.\sigma^{-n+2}(p) b_{n-2}(H)\right)+X^{n-1} \sigma^{-n+1}(p) b_{n-1}(H)$, где видпоследнего элемента станет каноническим, если взять остаток при делении каждого коэффициента на $\sigma(p)$. Рассматривая коэффициент при $X^{n-1}$, получаем $\sigma(p) \mid \sigma^{-n+1}(p) b_{n-1}(H)$, откуда $\sigma(p) \mid b_{n-1}(H)$ и $b_{n-1}(H)=0$. Аналогично получаем $b_{n-2}(H)=\cdots=b_{0}(H)=0$ и $f=0$.

Итак, $\operatorname{Hom}\left(V_{\alpha}, M\right) \cong \operatorname{Ext}\left(M_{\alpha}, M\right)$, поэтому достаточно доказать, что $\operatorname{Hom}\left(V_{\alpha}, M\right) \neq 0$. Но модуль $V_{\alpha}=A / A(Y, \sigma(p))$, а по замечанию выше $M=$ $A / A\left(Y, X^{n}, \sigma(p)\right)$, откуда вытекает требуемое.

Иначе, поскольку число конечных полуинтервалов на любой вырожденной орбите не более двух, модуль $M$ отвечает самому правому полуинтервалу $\left(\sigma^{-n}(p) ; p\right]$. Теперь проводим симметричную конструкцию с элементами $p_{\alpha}=\alpha X+p(H)$.

ГИПОТЕЗА. Пусть А обобщенная алгебра Вейля без ииклических орбит, $p(H)$ неразложим $и \sigma^{n}(p)$ делит $a(H)$ для по крайне мере 4 различных $n \in \mathbb{Z}$. Тогда конечномерный модуль $M$, соотвествующий внутреннему полуинтервалу, является началом левого почти расщепляющегося морфизма в категории модулей конечной длины над $A$.

СлЕдСтвИЕ. Пусть $\mathbf{k}$ алгебраически замкнутое поле характеристики нуль. Тогда ни один простой модуль над $\mathrm{Sl}_{2}(\mathbf{k})$ не является началом левого почти расщепляющегося морфизма в категории модулей конечной длины над $U_{\mathrm{sl}_{2}}(\mathbf{k})$.

ДокАЗАТЕЛЬСТво. Пусть $M$ - простой модуль над $U \mathrm{sl}_{2}(\mathbf{k})$. Тогда элемент Казимира $C$ действует на $M$ скалярно, т.е. $M$ простой модуль над ОАВ $A_{\lambda}=$ 
$U \mathrm{sl}_{2}(\mathbf{k}) / U \mathrm{sl}_{2}(\mathbf{k})(C-\lambda)$ с $\sigma(H)=H-1$ и $a(H)=\lambda-H(H+1)$. Поскольку $a(H)$ имеет не более двух корней, не существует ни одного левого п.р.м. в категории модулей конечной длины над $A_{\lambda}$, в частности, $M$ не инъективен над $A_{\lambda}$. Поэтому если $f: M \rightarrow N$ левый п.р.м. над $U \mathrm{sl}_{2}(\mathbf{k})$ и $M$ прост, то $f$ - вложение. Разложим $N=K \oplus L$, где $K$ - сумма подмодулей $N$ веса $\lambda$ и $L$ - сумма подмодулей $N$ других весов. Тогда $f(M) \subseteq K$ и $f: M \rightarrow K$ левый п.р.м. в категории модулей конечной длины над $A_{\lambda}$, что невозможно.

\section{СПИСОК ЦИТИРОВАННОЙ ЛИТЕРАТУРЫ}

[1] Бавула В. Обобщенные алгебры Вейля и их представления // Алгебра и анализ. 1992. Т. 4. № 1. C. $75-97$.

[2] Bavula V., van Oystaeyen F. The simple modules of certain generalized crossed products // J. Algebra. 1997. V. 194. № 2. P. 521-566.

[3] Zimmermann W. Auslander-Reiten sequences over derivation polynomial rings // J. Pure Appl. Algebra. 1991. V. 74. P. 317-332.

[4] Prest M., Puninski G. Some model theory over hereditary noetherian domains // J. Algebra. 1999. V. 211. P. 268-297.

[5] Bavula V. The extension group Ext of the simple modules over the first Weyl algebra // Proc. Lond. Math. Soc. (to appear). 